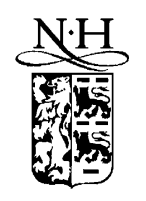

ELSEVIER

\title{
VUV spectroscopy of wide band-gap crystals doped with rare earth ions
}

\author{
V.N. Makhov ${ }^{\mathrm{a}, *}$, N.M. Khaidukov ${ }^{\mathrm{b}}$, N.Yu. Kirikova ${ }^{\mathrm{a}}$, M. Kirm ${ }^{\mathrm{c}}$, J.C. Krupa ${ }^{\mathrm{d}}$, \\ T.V. Ouvarova ${ }^{\mathrm{e}}$, G. Zimmerer ${ }^{\mathrm{c}}$ \\ ${ }^{a}$ Lebedev Physical Institute, Leninsky Pr. 53, 117924 Moscow, Russia \\ ${ }^{\mathrm{b}}$ Institute of General and Inorganic Chemistry, 117907 Moscow, Russia \\ ${ }^{\mathrm{c}}$ II. Institut für Experimentalphysik der Universität Hamburg, 22761 Hamburg, Germany \\ ${ }^{\mathrm{d}}$ Institut de Physique Nucleaire, 91406 Orsay Cedex, France \\ ${ }^{\mathrm{e}}$ General Physics Institute, 117942 Moscow, Russia
}

\begin{abstract}
Spectral and kinetic properties of vacuum ultraviolet (VUV) luminescence from different fluoride crystals doped with $\mathrm{Nd}^{3+}, \mathrm{Er}^{3+}$ or $\mathrm{Tm}^{3+}$ are discussed. Concentration quenching of VUV luminescence, the impact mechanism of the energy transfer from the matrix to the emission centers as well as nonradiative relaxation processes in the rare earth ions have been detected and studied. The crystal properties are analyzed from the viewpoint of their possible applications as materials for fast VUV scintillators and VUV solid state lasers. Only some of $\mathrm{Nd}^{3+}$ doped crystals possess simultaneously both high light output and relatively good timing properties of VUV luminescence and such crystals can be considered as promising materials for fast VUV scintillators. On the other hand, many of $\mathrm{Er}^{3+}$ and $\mathrm{Tm}^{3+}$ doped fluoride crystals are very attractive candidates as active media for tunable VUV solid state lasers with optical pumping. (C) 2001 Elsevier Science B.V. All rights reserved.
\end{abstract}

PACS: $78.55 ; 78.60 ; 78.40 ; 78.47 ; 42.70 . \mathrm{H}$

Keywords: Fluoride crystals; Rare earth ions; 5d-4f luminescence; VUV lasers; Scintillator

\section{Introduction}

The development of new efficient solid-state emitters in the vacuum ultraviolet (VUV) spectral range is an important and promising field of research for both scintillation and laser techniques. To move towards the development of new VUV scintillators and VUV solid state lasers, it is necessary first of all to characterize spectroscopic

\footnotetext{
*Corresponding author. Fax: +7-95-938-2251.

E-mail address: makhov@sci.lebedev.ru (V.N. Makhov).
}

properties of various combinations of wide bandgap hosts and doping ions displaying optical activity in the VUV spectral range. In this context, wide band-gap fluoride crystals doped with some trivalent rare earth (RE) ions with parity-allowed interconfiguration $5 \mathrm{~d}-4 \mathrm{f}$ radiative transitions, for example $\mathrm{Nd}^{3+}, \mathrm{Er}^{3+}$ and $\mathrm{Tm}^{3+}$, can offer the exceptional possibility for designing new VUV emitting materials [1].

In the present paper appropriate spectroscopic properties of some wide band-gap crystals doped with $\mathrm{Nd}^{3+}, \mathrm{Er}^{3+}$ or $\mathrm{Tm}^{3+}$ have been studied and 
analyzed from the viewpoint of their possible applications as materials for fast VUV scintillators and as active media for VUV solid state lasers.

\section{Experiment}

The studies of emission and excitation spectra as well as luminescence decay kinetics were performed at the SUPERLUMI station of HASY$\mathrm{LAB}$ under the excitation by the $5-30 \mathrm{eV}$ synchrotron radiation from the DORIS storage ring at DESY (Hamburg) [2]. The VUV luminescence was detected with a $0.5 \mathrm{~m}$ Pouey-type secondary VUV monochromator (with an instrumental resolution of about $1 \mathrm{~nm}$ ) equipped with a solar-blind photomultiplier for spectral measurements and a microsphere plate detector for measurements of decay curves. The time correlated single-photon counting was used for the measurements of time-resolved spectra and luminescence decay curves with a time resolution of $0.3 \mathrm{~ns}$.

Single crystals of different fluorides doped with $\mathrm{Nd}^{3+}, \mathrm{Er}^{3+}$ or $\mathrm{Tm}^{3+}$ were grown by using the vertical Bridgman [3] and hydrothermal synthesis techniques [4].

\section{Results and discussion}

The VUV luminescence of $\mathrm{Nd}^{3+}, \mathrm{Er}^{3+}$ or $\mathrm{Tm}^{3+}$ ions doped into wide band-gap crystals is due to interconfiguration transitions from the lowest level of the $4 \mathrm{f}^{n-1} 5 \mathrm{~d}$ configuration to different levels near the ground state of the $4 \mathrm{f}^{n}$ configuration [5]. The luminescence spectrum of a $\mathrm{LiKYF}_{5}$ crystal doped with $\mathrm{Nd}^{3+}$ is shown in Fig. 1. The broadband luminescence in the spectrum corresponds to interconfiguration $4 \mathrm{f}^{2} 5 \mathrm{~d}-4 \mathrm{f}^{3}$ transitions while the narrow lines observed at $\lambda>200 \mathrm{~nm}$ are due to intraconfiguration $4 \mathrm{f}^{3}-4 \mathrm{f}^{3}$ transitions in the $\mathrm{Nd}^{3+}$ ion (the assignments of emission bands to particular transitions are shown in the figure). Since $5 \mathrm{~d}-4 \mathrm{f}$ transitions are parity-allowed the decay times of $d-f$ luminescence should lie in the nanosecond range and, in particular, the decay time of $5 \mathrm{~d}-4 \mathrm{f}$ luminescence from $\mathrm{Nd}^{3+}$ in $\mathrm{LiKYF}_{5}$ is $9.1 \mathrm{~ns}$. However, in the case of $\mathrm{Er}^{3+}$ and $\mathrm{Tm}^{3+}$

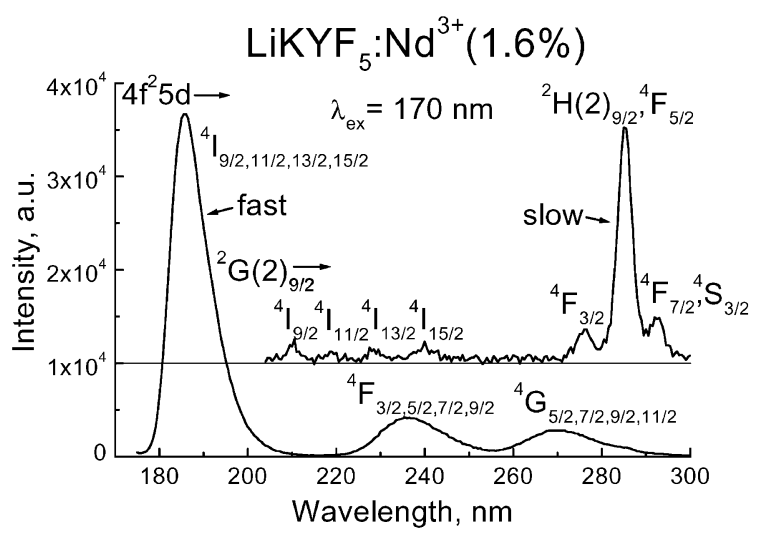

Fig. 1. Emission spectra of $5 \mathrm{~d}-4 \mathrm{f}$ and $4 \mathrm{f}-4 \mathrm{f}$ luminescence from $\mathrm{Nd}^{3+}$ doped into a $\mathrm{LiKYF}_{5}$ crystal excited by $170 \mathrm{~nm}$ photons at $300 \mathrm{~K}$. The assignments of the bands to particular transitions are shown.

the decay kinetics of $\mathrm{d}-\mathrm{f}$ luminescence is more complicated.

Recent studies of VUV emission of $\mathrm{Er}^{3+}$ and $\mathrm{Tm}^{3+}$ ions incorporated in some of fluoride crystals have shown that the emission spectrum due to interconfiguration $5 \mathrm{~d}-4 \mathrm{f}$ transitions consists of two series of bands for either of these ions: the fast emission bands with the decay constant in the range from a few to tens of nanoseconds for different crystals and (or) slow emission bands shifted to longer wavelengths with the decay constant in the microsecond range [6,7]. As it has been established [8], the fast and slow components arise from spin-allowed (S-A) and spin-forbidden (S-F) 5d-4f transitions, respectively. This effect can be illustrated by the luminescence spectra of a $\mathrm{BaY}_{2} \mathrm{~F}_{8}$ crystal doped with $\mathrm{Er}^{3+}$ and a $\mathrm{TmF}_{3}$ crystal (Figs. 2 and 3). The assignments of emission bands to various $5 \mathrm{~d}-4 \mathrm{f}$ transitions in $\mathrm{Er}^{3+}$ and $\mathrm{Tm}^{3+}$ are also shown in Figs. 2 and 3. The bands indicated in the figures correspond to the S-A $5 \mathrm{~d}-4 \mathrm{f}$ transitions (the fast component), i.e. transitions from the low-spin emitting state of the 4 f5d configuration $\left(2 \mathrm{~S}+1=4\right.$ for $\mathrm{Er}^{3+}$ and 3 for $\mathrm{Tm}^{3+}$ ) to different energy levels of the $4 \mathrm{f}$ ground configuration for $\mathrm{Er}^{3+}$ or $\mathrm{Tm}^{3+}$ ions. Accordingly, the bands due to the S-F $5 \mathrm{~d}-4 \mathrm{f}$ transitions (the slow component), i.e. transitions from the high-spin emitting state of the $4 \mathrm{f} 5 \mathrm{~d}$ 


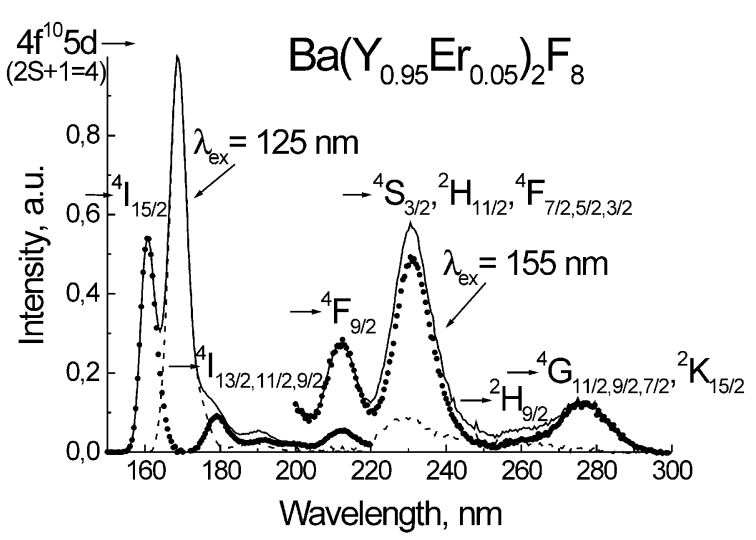

Fig. 2. Emission spectra of $5 \mathrm{~d}-4 \mathrm{f}$ luminescence from $\mathrm{Er}^{3+}$ doped into a $\mathrm{BaY}_{2} \mathrm{~F}_{8}$ crystal at $300 \mathrm{~K}$ (the dots indicate the fast component, the dashed line is the slow component and the solid line is the total intensity). The excitation wavelengths were 125 and $155 \mathrm{~nm}$ for different parts of the spectrum. The assignments of the bands to particular spin-allowed $5 \mathrm{~d}-4 \mathrm{f}$ transitions are shown.

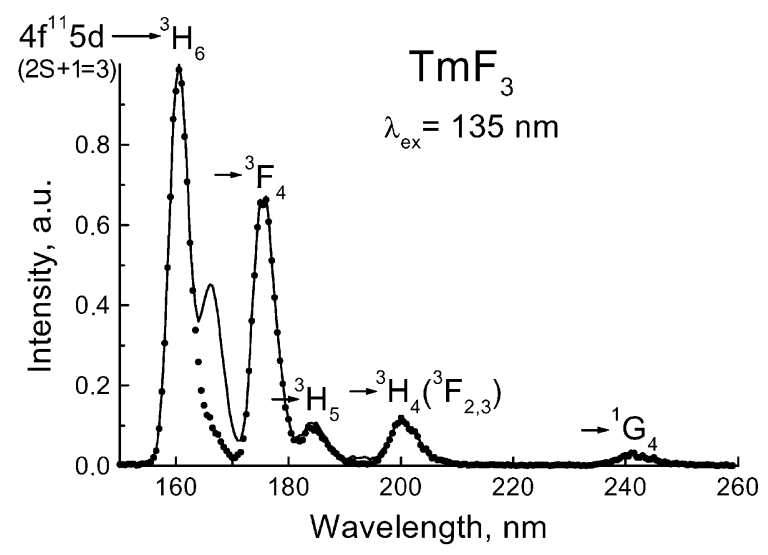

Fig. 3. Emission spectra of $5 \mathrm{~d}-4 \mathrm{f}$ luminescence from $\mathrm{Tm}^{3+}$ in a $\mathrm{TmF}_{3}$ crystal at $300 \mathrm{~K}$ (the dots indicate the fast component and the solid line is the total intensity). The excitation wavelength was $135 \mathrm{~nm}$. The assignments of the bands to particular spin-allowed $5 \mathrm{~d}-4 \mathrm{f}$ transitions are shown.

configuration $\left(2 \mathrm{~S}+1=6\right.$ for $\mathrm{Er}^{3+}$ and 5 for $\left.\mathrm{Tm}^{3+}\right)$ to the same energy levels of the $4 \mathrm{f}$ ground configuration for $\mathrm{Er}^{3+}$ or $\mathrm{Tm}^{3+}$, are shifted to longer wavelengths.

Excitation spectra of different emissions for $\mathrm{Er}^{3+}$ doped into a $\mathrm{LiYF}_{4}$ crystal are shown in Fig. 4. In the UV and the visible range only

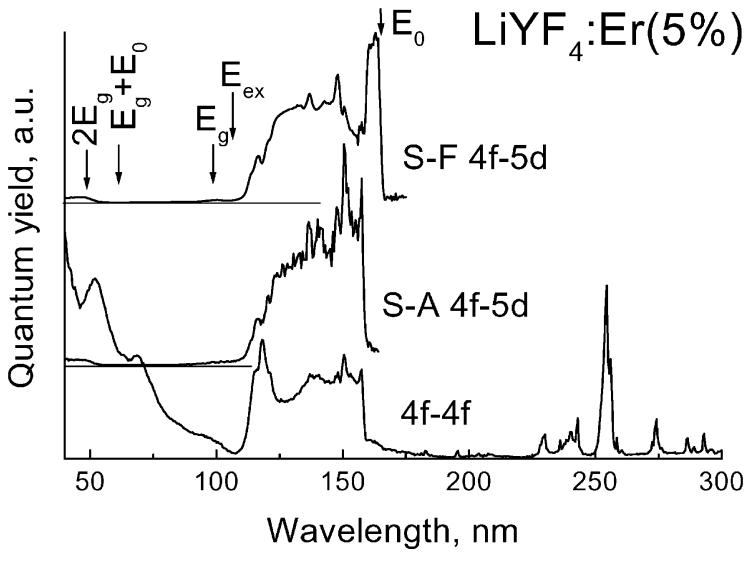

Fig. 4. Excitation spectra of the spin-allowed and the spinforbidden $5 \mathrm{~d}-4 \mathrm{f}$ luminescence as well as of the $4 \mathrm{f}-4 \mathrm{f}$ luminescence from $\mathrm{Er}^{3+}$ for a $5 \mathrm{at} \% \mathrm{Er}^{3+}$ doped $\mathrm{LiYF}_{4}$ crystal at $300 \mathrm{~K}$.

narrow lines in the excitation spectrum of $4 \mathrm{f}-4 \mathrm{f}$ luminescence are observed. At shorter wavelengths the onset of the S-F f-d absorption is situated at $\approx 165 \mathrm{~nm}$, which corresponds to the threshold of excitation for the S-F d-f microsecond luminescence. At even more shorter wavelengths the onset of the S-A $\mathrm{f}-\mathrm{d}$ absorption appears at $\sim 158 \mathrm{~nm}$. At these wavelengths the intensity of the S-F luminescence decreases sharply due to a higher value of the absorption coefficient for the S-A transitions. Actually, in this region the S-F luminescence is excited indirectly via the energy transfer from a higher-lying 5d level, from which the S-A transitions occur. The $\mathrm{f}-\mathrm{f}$ luminescence is also excited under both the S-F and the S-A f-d absorption. When the wavelength of excitation is close to the fundamental absorption edge of the $\mathrm{LiYF}_{4}$ matrix $(\lambda \sim 110 \mathrm{~nm})$ the quantum yield of both the S-A and the S-F d-f luminescence drops drastically although this is not observed in the case of the $f-f$ luminescence. In other words, no efficient energy conversion from the matrix to $\mathrm{d}-\mathrm{f}$ luminescence is observed for $\mathrm{Er}^{3+}$ and $\mathrm{Tm}^{3+}$ in both $\mathrm{LiYF}_{4}$ and most of other hosts. The efficiency of energy conversion is extremely low until the energy of excitation reaches the threshold for the processes of electronic excitations multiplication. This threshold $(\lambda \sim 55 \mathrm{~nm})$ has been found to lie at photon energy less than 2 band-gap energies and 
the effect is much more pronounced at higher concentrations of $\mathrm{Er}^{3+}$ or $\mathrm{Tm}^{3+}$ [9]. These features indicate clearly that the mechanism of the energy transfer from the matrix to $\mathrm{d}-\mathrm{f}$ luminescence is the so-called impact mechanism of electronic excitations multiplication when fast photoelectrons in the conduction band excite directly the emission centers in the crystals.

It has been found that stoichiometric $\mathrm{Er}^{3+}$ containing compounds have decay properties of $\mathrm{d}-\mathrm{f}$ luminescence which are absolutely different from those of stoichiometric $\mathrm{Tm}^{3+}$-containing crystals [10,11]: the decay constant of the S-F luminescence for $\mathrm{Er}^{3+}$ becomes as short as few nanoseconds while that for $\mathrm{Tm}^{3+}$ remains in the time range of hundreds of nanoseconds. This is due to the considerable difference between the energy level schemes for high-lying $4 f$ states of $\mathrm{Er}^{3+}$ and $\mathrm{Tm}^{3+}$. In case of $\mathrm{Tm}^{3+}$ no high-lying $4 \mathrm{f}$ levels with energies close to the energy of the lowest (emitting) 5d levels exist, while in case of $\mathrm{Er}^{3+}$ energy levels of some high-lying $4 \mathrm{f}$ states are very close to the emitting $5 \mathrm{~d}$ levels. Therefore, the additional channels of nonradiative decay via energy transfer to neighbouring ions lead to shortening (concentration quenching) the emission for both the S-F and the S-A d-f luminescence. The effect of concentration quenching is illustrated by Fig. 5, where shortening of the decay time for the $\mathrm{S}-\mathrm{F}$ luminescence with the $\mathrm{Er}^{3+}$ concentration is shown for a series of the $\mathrm{Ba}\left(\mathrm{Y}_{1-x} \mathrm{Er}_{x}\right)_{2} \mathrm{~F}_{8}$ crystals.

The existence of the slow S-F 5d-4f luminescence as well as very low efficiency of the energy transfer from the matrix to $5 \mathrm{~d}-4 \mathrm{f}$ luminescence make almost impossible the use of $\mathrm{Er}^{3+}$ and $\mathrm{Tm}^{3+}$ doped crystals as fast VUV scintillators. However, for $\mathrm{Er}^{3+}$ doped crystals the combined effect of concentration quenching and impact excitation can result in both fast decay and relatively high quantum yield of the $\mathrm{Er}^{3+} \mathrm{d}-\mathrm{f}$ luminescence in highly doped (stoichiometric) crystals. For example, at high-energy excitation in $\mathrm{Er}^{3+}$ doped $\mathrm{LiYF}_{4}$ crystals the concentration quenching leads to the decrease of the total quantum yield of $\mathrm{d}-\mathrm{f}$ luminescence, however the quantum yield of the fast S-A component $(\lambda \sim 157 \mathrm{~nm})$ increases with the $\mathrm{Er}^{3+}$ concentration [9]. Nevertheless,

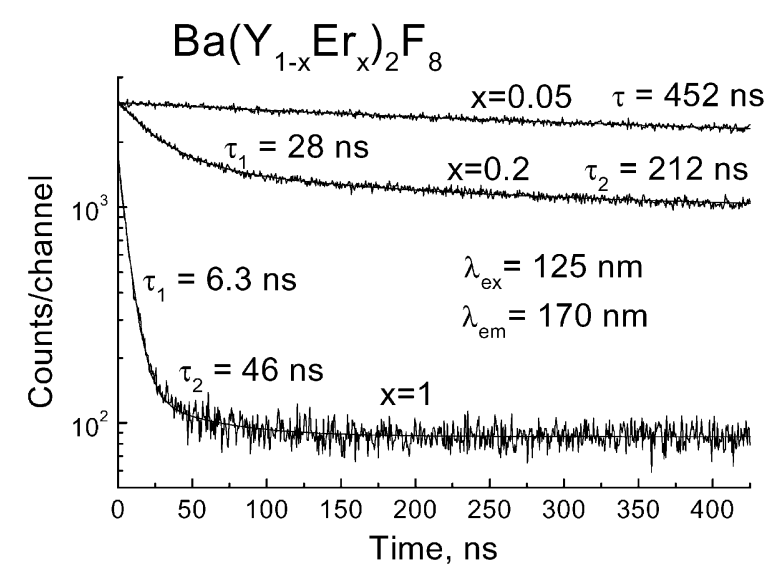

Fig. 5. Decay curves of the spin-forbidden $5 \mathrm{~d}-4 \mathrm{f}$ luminescence from $\mathrm{Er}^{3+}$ in $\mathrm{Ba}\left(\mathrm{Y}_{1-x} \mathrm{Er}_{x}\right)_{2} \mathrm{~F}_{8}$ crystals $(x=0.05 ; 0.2$ and 1.0) at $300 \mathrm{~K}$. The excitation wavelength was $125 \mathrm{~nm}$.

none of $\mathrm{Er}^{3+}$ or $\mathrm{Tm}^{3+}$ containing fluoride crystals studied in this work has VUV light output larger than that of the well-known scintillator $\mathrm{BaF}_{2}$.

The low light output of $d-f$ luminescence at high energy excitation is rather general property of fluoride crystals doped with RE ions. This is due to the fact that the conventional recombination mechanism of the energy transfer is usually inefficient in such crystals. However, in most oxygen-containing matrices the recombination mechanism can be very efficient although the decay kinetics usually has a considerable fraction of the slow component. In this context, two oxygen-containing matrices doped with $\mathrm{Nd}^{3+}$, namely $\mathrm{YPO}_{4}$ and $\mathrm{LuPO}_{4}$, where $\mathrm{d}-\mathrm{f}$ luminescence of $\mathrm{Nd}^{3+}$ in the VUV $(189 \mathrm{~nm})$ is observed, can be mentioned [12]. The light output of the VUV emission from these materials is comparable with that of most efficient $\mathrm{Ce}^{3+}$ doped scintillators, but timing properties of VUV emission are quite poor to obtain a good time resolution. On the other hand, at least one fluoride crystal, $\mathrm{K}_{2} \mathrm{YF}_{5}$, doped with $\mathrm{Nd}^{3+}$ in which the efficient recombination mechanism of the energy transfer from the matrix to $\mathrm{Nd}^{3+}$ resulting in $5 \mathrm{~d}-4 \mathrm{f}$ luminescence $(185 \mathrm{~nm})$ is observed has been found for the present time [11]. Besides, it is not unlikely to suppress considerably the slow component of the $5 d-4 f$ 
luminescence by a proper choice of the RE ion concentration.

The competition between the S-A and the S-F luminescence of $\mathrm{Er}^{3+}$ or $\mathrm{Tm}^{3+}$ depends on the relative energy position of emitting $5 \mathrm{~d}$ levels original for the S-A and the S-F transitions. If the energy gap between these levels is small enough (not more than 4-5 times larger than the cutoff phonon energy of the crystal), multi-phonon relaxation will exceed the rate of the radiative decay from the higher-lying $5 \mathrm{~d}$ level responsible for the S-A transitions, which results in "pumping" the lower-lying "metastable" 5d level responsible for the $\mathrm{S}-\mathrm{F}$ transitions. In other words, the energy level diagram of the $5 \mathrm{~d}-4 \mathrm{f}$ transitions for these two ions in fluoride crystals represents a typical four-level laser scheme. Indeed, in some crystals, e.g. $\mathrm{KYF}_{4}$, the intensity of the S-A luminescence of $\mathrm{Er}^{3+}$ or $\mathrm{Tm}^{3+}$ is extremely weak [13], i.e. in such crystals there exists very efficient nonradiative relaxation from higher-lying $5 \mathrm{~d}$ states to the lowest $5 \mathrm{~d}$ level responsible for the S-F luminescence. This peculiarity results in a larger Stokes shift of $5 \mathrm{~d}-4 \mathrm{f}$ luminescence from the edge of the strong S-A $4 \mathrm{f}-5 \mathrm{~d}$ absorption for $\mathrm{Er}^{3+}$ and $\mathrm{Tm}^{3+}$ than for $\mathrm{Nd}^{3+}$, i.e. to a smaller reabsorption of emitted radiation by such crystals. Relatively wide emission bands due to $5 \mathrm{~d}-4 \mathrm{f}$ transitions in these ions (for example, in a $\mathrm{KYF}_{4}$ crystal the FWHM is 4.6 and $6.5 \mathrm{~nm}$ for $\mathrm{Er}^{3+}$ and $\mathrm{Tm}^{3+}$, respectively) offer a possibility for tunable laser design. Obviously, one can expect that optical pumping, for example from a noble-gas discharge, will be very efficient in the wide spectral range of strong $4 \mathrm{f}-5 \mathrm{~d}$ absorption.

\section{Conclusions}

It has been shown that many fluoride crystals doped with $\mathrm{Nd}^{3+}, \mathrm{Er}^{3+}$ or $\mathrm{Tm}^{3+}$ have intense VUV luminescence due to interconfiguration $5 \mathrm{~d}-4 \mathrm{f}$ transitions in the RE ions. Strong concentration quenching, which leads to nanosecond decay of the S-F 5d-4f luminescence from $\mathrm{Er}^{3+}$, has been found in crystals highly doped with $\mathrm{Er}^{3+}$. Due to this feature the decay properties of VUV lumines- cence from $\mathrm{Er}^{3+}$ and $\mathrm{Tm}^{3+}$ in highly doped fluoride crystals are absolutely different.

Neither $\mathrm{Er}^{3+}$ - nor $\mathrm{Tm}^{3+}$-containing materials studied in this work have shown better scintillation properties than those of the well-known scintillator $\mathrm{BaF}_{2}$ for the use as fast VUV scintillators. Only some $\mathrm{Nd}^{3+}$ doped crystals have simultaneously both high light output and relatively good timing properties of VUV luminescence.

The S-A $5 \mathrm{~d}-4 \mathrm{f}$ luminescence of $\mathrm{Er}^{3+}$ or $\mathrm{Tm}^{3+}$ doped into some crystals is extremely weak, i.e. in such crystals there exists very efficient nonradiative relaxation from higher-lying $5 \mathrm{~d}$ states to the lowest $5 \mathrm{~d}$ level responsible for the $\mathrm{S}-\mathrm{F}$ luminescence. This peculiarity of the studied crystals doped with $\mathrm{Er}^{3+}$ or $\mathrm{Tm}^{3+}$ can be very attractive for developing VUV solid state lasers based on crystals of such a type.

\section{Acknowledgements}

The support of INTAS Grant 99-01350, Graduiertenkolleg Fields and localized atomsAtoms and localized fields: Spectroscopy of localized atomic systems and Federal Program "Integration" is gratefully acknowledged.

\section{References}

[1] J.C. Krupa, et al., Mater. Sci. Forum 315-317 (1999) 27.

[2] G. Zimmerer, Nucl. Instr. and Meth. A 308 (1991) 178.

[3] A.A. Kaminskii, T.V. Ouvarova, Izv. Akad. Nauk SSSR, Ser. Neorg. Mat. 24 (1980) 2080.

[4] M.A. Dubinskii, et al., J. Modern Opt. 37 (1990) 1355.

[5] K.H. Yang, J.A. DeLuca, Appl. Phys. Lett. 29 (1976) 499.

[6] V.N. Makhov, et al., HASYLAB Annual Report 1996, Part I, p. 273.

[7] J. Becker, et al., J. Lumin. 78 (1998) 91.

[8] R.T. Wegh, H. Donker, A. Meijerink, Phys. Rev. B 57 (1998) R2025.

[9] V.N. Makhov, et al., J. Lumin. 87-89 (2000) 1005.

[10] J.Y. Gesland, et al., J. Electron Spectrosc. Relat. Phenom. 101-103 (1999) 579.

[11] V.N. Makhov, et al., Proc. 5th Int. Conf. on Inorganic Scintillators and their Applications SCINT'99, Moscow, August 16-20, 1999. p. 369.

[12] J. Becker, et al., J. Alloys Compounds 275-277 (1998) 205.

[13] N.M. Khaidukov, G. Zimmerer, et al., Opt. Commun. 184 (2000) 183. 